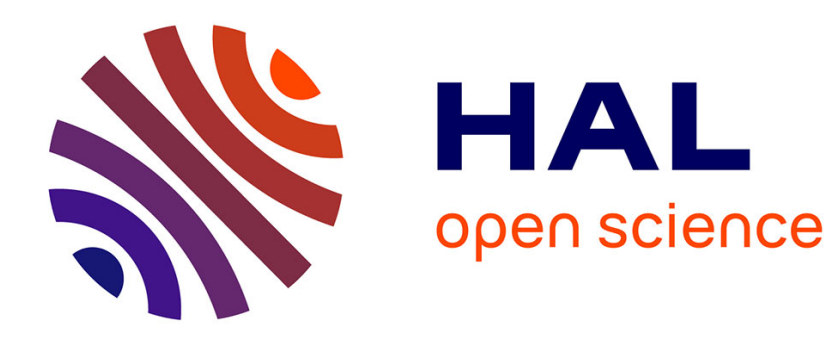

\title{
Josephson junctions as resonant detectors
}

C.D. Andriesse

\section{To cite this version:}

C.D. Andriesse. Josephson junctions as resonant detectors. Revue de Physique Appliquée, 1974, 9

(1), pp.119-123. 10.1051/rphysap:0197400901011900 . jpa-00243720

\section{HAL Id: jpa-00243720 \\ https://hal.science/jpa-00243720}

Submitted on 1 Jan 1974

HAL is a multi-disciplinary open access archive for the deposit and dissemination of scientific research documents, whether they are published or not. The documents may come from teaching and research institutions in France or abroad, or from public or private research centers.
L'archive ouverte pluridisciplinaire HAL, est destinée au dépôt et à la diffusion de documents scientifiques de niveau recherche, publiés ou non, émanant des établissements d'enseignement et de recherche français ou étrangers, des laboratoires publics ou privés. 


\title{
JOSEPHSON JUNCTIONS AS RESONANT DETECTORS
}

\author{
C. D. ANDRIESSE
}

\author{
Kapteyn Astronomical Institute, Department of Space Research \\ University of Groningen, The Netherlands
}

\begin{abstract}
Résumé. - Nous étudions la résonance d'une cavité couplée à une jonction Josephson correctement polarisée et soumise à un champ à la fréquence de résonance. Nous décrivons une cavité Fabry-Pérot accordable, mettant en jeu un dispositif à contact à pointe, qui peut être utilisée comme détecteur sélectif d'un rayonnement infrarouge lointain. Des données expérimentales préliminaires montrent que le principe est correct, mais que le couplage entre la jonction et la cavité est encore trop faible d'un ordre de grandeur. Des modifications qui amélioreront les performances sont décrites.

Abstract. - The single resonance is treated of a cavity, which is driven by an appropriately biased Josephson junction and an external field at resonance frequency. We describe a tunable Fabry-Pérot cavity, based on a flat point-contact device, as a possible narrow-band detector of far-infrared radiation. Preliminary experimental data for this system indicate that the principle works, but that the coupling of tunnel currents with the resonance still falls short by an order of magnitude. Modifications are discussed that will improve its performance.
\end{abstract}

1. Introduction. - Astronomical observations of line radiation from atoms and molecules at farinfrared wavelengths $(0.1-3 \mathrm{~mm})$ will be of great value for understanding physical processes in the interstellar medium. They probably will shed more light on the cooling of this medium [1] and may turn out crucial for the knowledge of regions which strongly emit in the far infrared [2]. For such observations detection systems are required with low noise and high spectral resolution, competitive if possible with that of radio heterodyne systems. This may explain why astronomers are interested in the development of Josephson junction devices, which for this wavelength range might meet both requirements [3].

In this paper an account is given of work at the Kapteyn Astronomical Institute on a tunable resonant detector excited by a Josephson point-contact junction. The basic idea for this device has been discussed by Richards and Sterling [4]. If operated at an appropriate voltage bias the junction currents can be coupled easily to the resonance field of a cavity, so that a current step is induced. Any field entering the cavity will affect the coupling of the currents with the resonance and detection then amounts to measuring the resultant height variation of the current step. The principal merit of a resonant detector is its small spectral bandwidth, which implies low noise.

In our device we tried to optimize the coupling between the cavity and the junction on the one hand and between the cavity and external fields on the other hand. At the same time we pursued tunability of the cavity over a wide range. These goals may still be remote, but a description of our approach might have some value. We begin with a short discussion of calculations on the resonant detector.

2. Calculations. - As is well known [5], ac tunnel currents appear in any weak link of two superconductors when a voltage bias is applied across the junction. To understand the interaction of far-infrared radiation with these currents, one has to solve the ac portion of Maxwell's equations subject to appropriate boundary conditions. This is a complicated nonlinear problem, which may turn out untractable for most experimental situations.

For the case of a point-contact junction at some position in a cavity one can simplify the problem by assuming that the tunnel currents radiate a dipole field characterized by junction dimension and normal resistance [6]. The extent to which each of the cavity modes overlaps with the dipole field then determines the coupling. In view of the relatively low impedance of point contacts, one can argue that as a rule the currents will only excite waves with electrical nodes close to the position of the junction. Evidence for this has been first given by Dayem and Grimes [7]. Nonlinearity of the junction, however, will cause mode coupling, so that energy may be stored in the many different modes that satisfy this rule.

For the simple geometry of oxide layer junctions Werthamer and Shapiro [8] assumed that the currents dominantly couple to a single mode of the cavity, here formed by the oxide layer itself. They base this on a geometrical analogy with lasers, for which a study of mode competition learns that only a single mode will be dominantly excited at any one 
moment. Such an argument might not hold for any geometry and the validity of single-mode coupling should be verified for each experimental configuration.

Werthamer and Shapiro further showed that solving Maxwell's equations for the currents in an oxide layer equals solving the differential equation for a damped harmonic oscillator, representing the cavity, which is driven by the tunnel currents. The coupling between cavity and junction is determined here by the ratio of the zero-voltage current and the capacity of the oxide layer. Generalization of this result to other junctions probably is allowed, but then a more general coupling constant $A$ has to be used. So

$$
\begin{aligned}
{\left[\frac{\mathrm{d}^{2}}{\mathrm{~d} t^{2}}+\gamma \frac{\mathrm{d}}{\mathrm{d} t}+\omega_{\mathrm{c}}^{2}\right] \Omega(t) } & = \\
= & A \frac{\mathrm{d}}{\mathrm{d} t} \cos \left[\Omega_{\mathrm{B}} t+\int^{t} \mathrm{~d} \tau \Omega(\tau)\right],
\end{aligned}
$$

where $\omega_{\mathrm{c}}$ is the mode frequency of the cavity, $\gamma$ its damping, $\Omega=2 \mathrm{e} V / \hbar$ (as usual $2 \mathrm{e}$ is the charge of a Cooper pair and $\hbar$ Planck's constant divided by $2 \pi$ ) and $V$ is a voltage bias $V_{\mathbf{B}}\left(\rightarrow \Omega_{\mathrm{B}}\right)$ across the junction cq a time dependent voltage $V(t)(\rightarrow \Omega(t))$ caused by the tunnel currents. If $\Omega_{\mathrm{B}}$ approaches $\omega_{\mathrm{c}}$ one gets resonance, in which case the frequency component of $\Omega(t)$ at $\Omega_{\mathrm{B}}$ will be predominant. Therefore it seems reasonable to approximate

$$
\int^{t} \mathrm{~d} \tau \Omega(\tau) \approx z \cos \left(\Omega_{\mathrm{B}} t+\varphi\right) .
$$

The amplitude $z$ and phase $\varphi$ now can be determined from the implicit algebraic equation, which in this case results from (1). For the height of the self-induced step at $\Omega_{\mathbf{B}}=\omega_{\mathrm{c}}$, normalized with the zero-voltage current $i_{0}$, then follows

$$
\frac{\Delta i}{i_{0}}=J_{1}(z)
$$

with an amplitude-coupling relation

$$
\frac{A}{\gamma \omega_{\mathrm{c}}}=\frac{z^{2}}{2 J_{1}(z)} .
$$

For small field amplitudes the Bessel function $J_{1}(z)=\frac{1}{2} z=A /\left(2 \gamma \omega_{\mathrm{c}}\right)$, so that the step develops proportional to $A$. The maximum height is obtained for $z=1.841$, where $J_{1}(z)=0.582$. However, to reach this height the coupling $A$ needs to be as high as $2.914 \gamma \omega_{\mathrm{c}}$, as follows from (4). Russer [9] has shown that the dependence (3) actually deviates somewhat from $J_{1}(z)$. Therefore the above numbers will be indicative rather than accurate.

We now turn to the consequences of an external field entering the cavity. At first sight it seems proper to add in (1) a term $\Omega_{\mathrm{e}} \cos \left(\omega_{\mathrm{e}} t+\varphi_{\mathrm{e}}\right)$ to $\Omega(t)$, which physically amounts to direct coupling of this field with the currents. Neglecting then the phase $\varphi_{\mathrm{e}}$, as it will be incoherent with the phase of the resonance, this leads to multiplication of (4) with the factor $J_{0}\left(\Omega_{\mathrm{e}} / \omega_{\mathrm{e}}\right)$ [8]. In effect the external field thus would enhance the coupling of the currents with the resonance, a result valid for any frequency $\omega_{\mathrm{e}}$.

However, one has to account for the phase $\varphi_{\mathrm{e}}$ more carefully. Furthermore Sterling [10] has argued that it is more satisfactory to introduce in (1) a second drive term of the cavity, viz. $B \mathrm{~d} / \mathrm{d} t z_{\mathrm{e}} \cos \left(\omega_{\mathrm{e}} t+\varphi_{\mathrm{e}}\right)$, $B$ being the coupling constant of the external field with the cavity and $z_{\mathrm{e}}$ the amplitude of the external field. This view is shared by Ulrich and Kluth [11] in their treatment of homodyne detection, but mathematically the problem remains very much the same. Making explicit use of $\omega_{\mathrm{e}}=\omega_{\mathrm{c}}$ and accounting for the phase difference $\left(\varphi_{\mathrm{e}}-\varphi\right)$, which incoherently contributes the time averages $\left\langle\cos ^{2}\left(\varphi_{\mathrm{e}}-\varphi\right)\right\rangle=\frac{1}{2}$, they find for the current step at $\Omega_{\mathrm{B}}=\omega_{\mathrm{c}}$

$$
\frac{\Delta i}{i_{0}}=\frac{J_{1}(z)}{\sqrt{1+\frac{1}{2} \sigma^{2}\left\{1+\left(J_{1}(z) / J_{1}^{\prime}(z)\right)^{2}\right\}}}
$$

with an amplitude-coupling relation

$$
\frac{A}{\gamma \omega_{\mathrm{c}}}=\frac{z^{2}}{2 J_{1}(z)} \sqrt{1+\frac{1}{2} \sigma^{2}\left\{1+\left(\frac{J_{1}(z)}{J_{1}^{\prime}(z)}\right)^{2}\right\}} .
$$

Here $\sigma$ represents the relative external field amplitude as coupled into the cavity, $\sigma=B z_{\mathrm{e}} /\left(\gamma \omega_{\mathrm{c}} z\right)$.

By comparing (6) and (4) it is evident that the presence of an external field effectively enhances the coupling. However, from (5) it follows that this leads to a decrease rather than an increase of the current step. In fact the tunnel currents and the external field are complementary in exciting the resonance : when the field increases, less current is needed to maintain it. A singularity occurs for the case discussed above that $J_{1}(z)=0.582$, where the derivative $J_{1}^{\prime}(z)=0$. Here the effective enhancement of $A$ becomes infinite, whereas at the same time the current step disappears. In the vicinity of this singularity $(z \approx 1.841)$, however, and as long as $\sigma$ is not too small (5), reads

$$
\frac{\Delta i}{i_{0}} \approx \sqrt{2} \frac{J_{1}^{\prime}(z)}{\sigma}
$$

showing a pathological response indeed.

There are many uncertainties in the derivation of the above formulae. A further analysis of the resonant case, where noise is included from the onset and due consideration is given to the single-mode coupling and approximation (2), might shed more light on the singular response. Note that a most efficient coupling $A$ is needed to observe such a response in practice (see also section 4).

Finally we mention that the spectral bandwidth, which is determined by the frequency shift $\left(\omega_{\mathrm{c}}-\Omega_{\mathrm{B}}\right)$ that halves the step height, is considerably less than given by the quality $Q=\omega_{\mathrm{c}} / \gamma$ of the cavity. This follows from a numerical treatment of (1) with the second drive term and (2) for the case that $\Omega_{\mathbf{B}}$ differs 
from $\omega_{\mathrm{c}}$. More convincing perhaps is the experimental verification of this "narrowing » by Richards and Sterling [4], who quote effective $Q^{\prime} s$ which exceed $\omega_{c} / \gamma$ by an order of magnitude or more. It is evident that this is of great importance for the noise in a resonant detector.

3. Resonant device. - A resonant detector requires good coupling of the tunnel currents with the cavity, to induce a clear current step, and of the external field with the cavity, to obtain a measurable modulation of the current step.

Consider the first coupling. We mentioned the rule that the currents will preferably couple to waves with electrical nodes close to the junction. When tunability of the cavity is pursued by changing dimensions, the node positions will change also, except for the ones at the surfaces. Furthermore the field associated with the currents overlaps principally with cavity modes propagating perpendicular to the currents. Therefore the currents should have large components in the plane of the surface, which can be obtained by directing a point-contact junction under a small angle with this plane. These considerations led to the device shown in figure 1 , which forms a wall for a cavity.

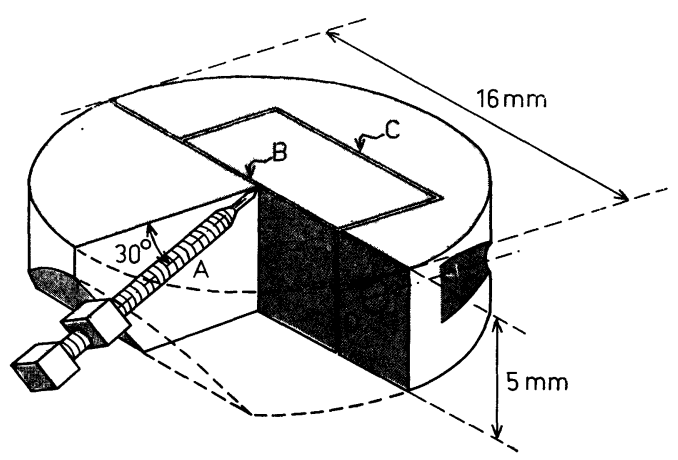

FIG. 1. - Drawing of the $\mathrm{Nb}$ point-contact device with cutaway quadrant. $\mathrm{A}=$ needle, $\mathbf{B}=$ position of mylar sheet, $\mathrm{C}=$ glass connection and $\mathrm{D}=$ screw position.

The device consists of two $\mathrm{Nb}$ blocks, bolted together by two $\mathrm{Nb}$ screws and separated by a $0.02 \mathrm{~mm}$ thick sheet of mylar, which is cut about $0.5 \mathrm{~mm}$ below the surface. In the one block an isolated section is introduced using a $0.2 \mathrm{~mm}$ thick glass connection of matched thermal expansion (Schott-Jena 101) with a resistance of $10^{5} \Omega$. This connection is made by induction melting of the glass powder under $\mathrm{Ar}$ pressure after rigorous high vacuum cleaning of the $\mathrm{Nb}$ surface at $1000^{\circ} \mathrm{C}$. In the other block a $1 \mathrm{~mm}$ shaft is bored under $30^{\circ}$ with the surface containing M 1 fine thread, which ends in a narrower conical section making a $0.05 \mathrm{~mm}$ diameter hole, $0.1 \mathrm{~mm}$ below the surface.

The above shaft tightly holds a $\mathrm{Nb}$ needle, provided with counter nut, which has a cone with full top angle of $30^{\circ}$ and with top-rounding radius of about $0.01 \mathrm{~mm}$. After machining the needle we etch it, as well as the opposing surface, in a $\mathrm{HNO}_{3}-\mathrm{HF}-\mathrm{CH}_{3} \mathrm{COOH}$ solution, thoroughly clean it and let it shortly (or not) oxidize in an $\mathrm{O}_{2}$ atmosphere. The contacts we made are characterized by normal shunt resistances $R$ of $0.5-2 \Omega$ and a capacity $C$ of about $15 \mathrm{pF}$, being determined by the dimensions of the above mentioned isolated section and mylar sheet. We use four leads, two for the current source (cq-measurement) and two for the voltage measurement (cq-bias). A typical current-voltage characteristic is shown in figure 2 .

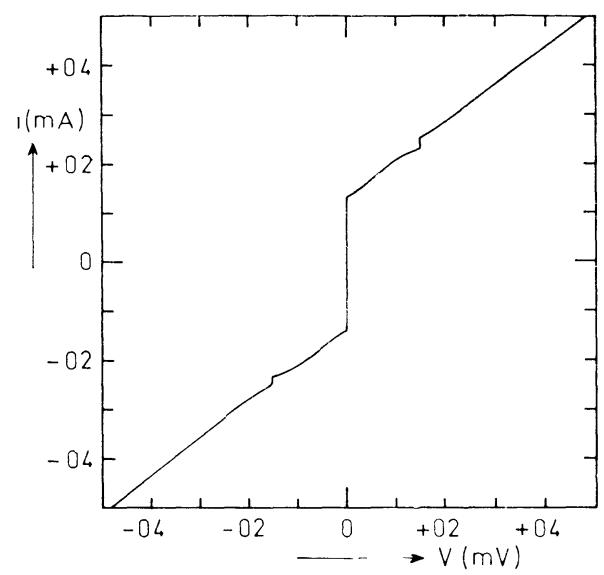

FIG. 2. - A current-voltage characteristic for the device shown in figure 1 at $4.4 \mathrm{~K}$ and free standing. The current steps of $0.02 \mathrm{~mA}$ at $\pm 0.145 \mathrm{mV}$ are due to a resonance in the supporting ring (see Fig. 3). The quantity $\beta_{\mathrm{c}}=2 \mathrm{e} i_{0} C R^{2} / \hbar$, defined by McCumber [12], is about $10\left(i_{0} \approx 0.25 \mathrm{~mA}, R \approx 0.8 \Omega\right)$. Inductive effects apparently are small.

Next consider the coupling of the external field with the cavity. We restrict ourselves to the Fabry-Pérot geometry of two parallel plane surfaces, the one formed by the above device and the other by a reflective plate. The cavity modes we are interested in are standing waves between these two surfaces, the wavelengths of which can be selected by adjusting the distance. Other modes, relevant for the fixed geometry in perpendicular directions, might be excited also, however. As is well known for Fabry-Pérot systems [13], high quality (= finesse) is obtained at the expense of coupling with an external field. The latter has to pass through the reflective plate, either by making small holes in it or by having it semitransparent. In the case of holes (e. g. in a metal mesh) one has a freedom in dimensions to find a suitable compromise between quality and transmission, but then these quantities will be wavelength dependent. In the case of a semitransparent plate (e. g. an intrinsic semiconductor) such a freedom does not exist - unless selective coatings are applied -, but independence can be obtained of the wavelength. These considerations led to the instrument sketched in figure 3 , to which we refer in the description below.

In this instrument the surface of the point-contact device $A$ is placed at a variable distance of a few $\mathrm{mm}$ 


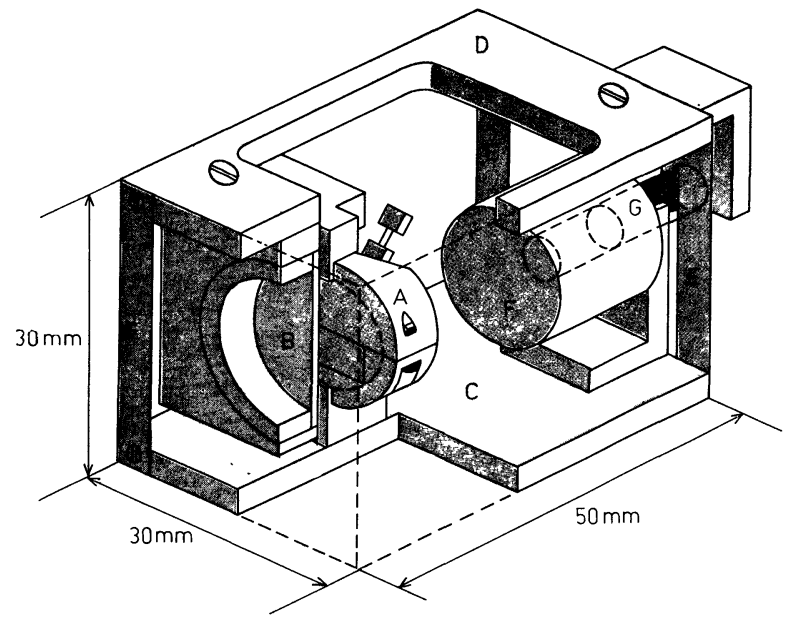

FIG. 3. - Drawing of the tunable resonator with cut-away section as explained in the text.

parallel to the surface of a slightly wedged, about $1 \mathrm{~mm}$ thick, Ge plate B. Parallel guiding is obtained by four leaf springs $\mathrm{E}$, which are attached to the lower plate $C$, carrying $A$, and the upper frame $D$, carrying $B$. The distance between $\mathrm{A}$ and $\mathrm{B}$ can be adjusted by varying the current in the $\mathrm{Nb}$ wire of coil $\mathrm{F}$, attached to $C$, which encloses part of the permanent magnet $G$, attached to D. Displacements of about $1 \mathrm{~mm}$ require a current of $0.5 \mathrm{~A}$, the magnetic field of which is shielded from A by a double $\mu$-metal envelope of the coil. In most point contacts no depression of the zerovoltage current was observed in the presence of currents in the coil.

The Ge plate, obtained from Quartz and Silice S. A., has a specific resistance exceeding $10 \Omega$.m and consequently shows no free-carrier absorption (we only observed the near-infrared phonon-absorption peaks). Neglecting therefore the imaginary index of refraction and adopting a real index $n=3.994$ [14], we obtain for the quality at normal indicence [13]

$$
Q=\left(n^{2}-1\right)^{2} / 4 n^{2}=3.504
$$

and for the transmission $4 n /(n+1)^{2}=0.6406$. The Ge plate was set parallel to A within $0.05 \mathrm{~mm}$ by pressing it against the supporting ring of $\mathrm{A}$ before fixing the holder to $\mathrm{D}$; $\mathrm{A}$ is pressed against the ring by three bent springs.

The above instrument is cooled in a LeyboldHeraeus liquid-helium flow cryostat to temperatures around $4 \mathrm{~K}$. External fields, chopped at $13 \mathrm{~s}^{-1}$, can enter through a window in front of the Ge plate. A $0.337 \mathrm{~mm}$ wavelength beam is available from a General Electrical Company $\mathrm{HCN}$ maser (NPL design) at adjustable power levels below a milliwatt. We apply a current source in sweep mode to obtain current-voltage characteristics and derivatives of them on an oscilloscope. At the moment a dedicated voltage source is built with an output impedance of $10^{-4} \Omega$ and a stability of $10^{-5}$. The current steps may be properly measured with an impedance transformer, the primary coil of which consists of $\mathrm{Nb}$ wire in series with the junction. Our data so far have been obtained with the current source.

4. Results. - Using the above instrumentation we started to study the position and the height of selfinduced current steps as a function of the distance between the point-contact device and the Ge window. Furthermore we try to measure the step response to broad-band as well as monochromatic $(0.337 \mathrm{~mm})$ radiation as a function of the incident power. By lack of a proper voltage source only preliminary results can be given here, which still do not cover the complete program.

In figure 4 we show part of a current-voltage characteristic obtained for a Fabry-Pérot distance of $8.0 \mathrm{~mm}$. In this case we have clear self-induced steps at $(78 \pm 10)$, $(196 \pm 10), \quad(250 \pm 10)$ and $(378 \pm 10) \mu \mathrm{V}$ with heights of $(22 \pm 8),(14 \pm 5),(8 \pm 3)$ and $(10 \pm 2) \mu \mathrm{A}$, respectively. In spite of the relatively low $\omega_{\mathrm{c}}$ the steps are quite small $\left(\Delta i / i_{0} \lesssim 0.05\right)$. So far we could not firmly establish that the step height grows with $\omega_{\mathrm{c}}^{-1}$, as follows from (3) for small amplitudes. By halving the Fabry-Pérot distance, the first step is shifted to the double voltage.

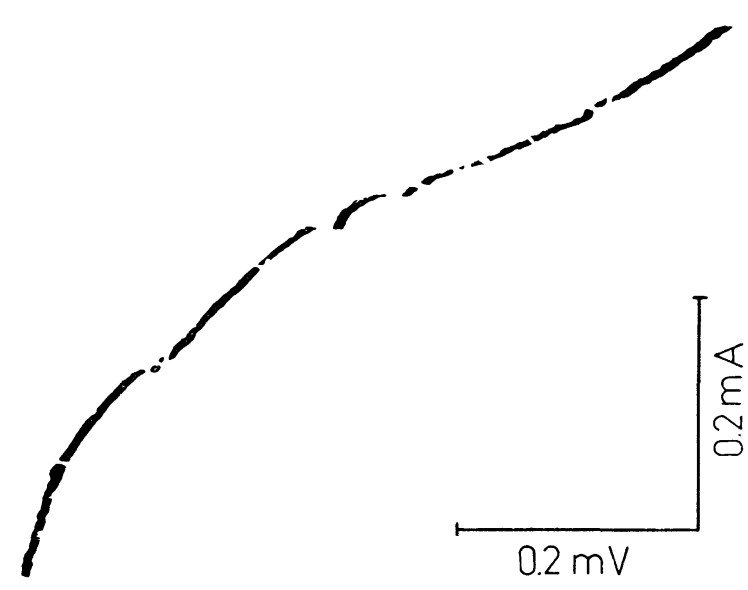

FIG. 4. - Negative photograph of oscilloscope trace of a current-voltage characteristic for the resonator shown. in figure 3. Broadening of the trace in voltage direction is due to noise on the junction signal. The upper end of the zero-voltage step is below, left. In the text the small self-induced current steps are discussed.

It is evident that the first step, which corresponds to the selected wavelength, is hardly higher than the other steps, which are no simple harmonics of the former and presumably correspond to independent resonances of the cavity. The finite slope of the first step, as well as its relatively small height, is explained by the low quality of the resonator for the selected mode. The greater sharpness of the other steps indicates that a much lower damping is involved, which compensates for the weaker coupling. These modes probably are due to the highly reflective supports of the pointcontact device and the Ge window, for which we intend 
to replace metal by a suitable isolator. Note that the mode excited in the free-standing point-contact device (Fig. 2) is not excited in the Fabry-Pérot arrangement.

The fact that the selected self-induced step is quite small means that the coupling constant $A$ is not yet satisfactory. For the above $8.0 \mathrm{~mm}$ distance case we find, from the calculated $Q$ and the geometrical value for $\omega_{\mathrm{c}}, A \lesssim 2 \times 10^{21} \mathrm{~s}^{-2}$. To obtain a singular response we have to increase the coupling by an order of magnitude. A possible way is to reduce the capacity of the point-contact device by increasing the distance « $B »$ (Fig. 1). Simple considerations show that the power in a resonance field for singular response should be as high as $10^{-9} \mathrm{~W}$. To excite such a field the matrix of weak links in a superconducting film covering a flat substrate, described by Mooij et al. [15], may be more suitable than our device.

So far no conspicuous effects on the step height were visible due to external radiation. In particular only a slight response was found to the maser line (about $25 \%$ reduction for a power of $10^{-5} \mathrm{~W}$ ). At the moment our electronics falls short to study the effects with adequate accuracy.

Though a complete evaluation is not yet possible, we conclude that our approach to a tunable resonant detector, based on a Josephson junction, is promising. Some modifications may enhance the coupling of the tunnel currents with a selected resonance sufficiently to obtain a singular response. We finally mention that in our set-up the point contact is very stable and not affected by activities like assembling and transport.

Acknowledgments. - The device has been skilfully made by H. H. A. Schaeffer, who also assisted in the measurements. Thanks are due to T. M. Klapwijk and J. E. Mooij for helpful comments.

\section{References}

[1] Dalgarno, A. and McCray, R. A., Annual Review of Astronomy and Astrophysics 10 (1972) 375.

[2] Neugebauer, G., Becklin, E. and Hyland, A. R., ibidem 9 (1971) 67.

[3] Ulrich, B. T., Proc. 12th Int. Conf. on Low Temperature Physics (Keigaku, Tokyo) 1970867.

[4] Richards, P. L. and Sterling, S. A., Appl. Phys. Lett. 14 (1969) 394.

[5] Anderson, P. W., Progress in Low Temperature Physics 5 (1967) 1.

[6] Zimmerman, J. E., J. Appl. Phys. 42 (1971) 30.

[7] Dayem, A. H. and Grimes, C. C., Appl. Phys. Lett. 9 (1966) 47.
[8] Werthamer, N. R. and Shapiro, S., Phys. Rev. 164 (1967) 523.

[9] Russer, P., Acta Phys. Austriaca 32 (1970) 373.

[10] Sterling, S. A., Thesis Berkeley 1970.

[11] Ulrich, B. T. and Kluth, E. O., Proc. IEEE 61 (1973) 51.

[12] McCumber, D. E., J. Appl. Phys. 39 (1968) 3113.

[13] Françon, M., Optical Interferometry (Academic Press) 1966.

[14] Moss, T. S., Optical properties of semiconductors (Butter worths) 1961.

[15] Moois, J. E., Gorter, C. A. and Noordam, J. E., Revue Phys. Appl. 9 (1974) 173. 